\title{
SKEW-SYMMETRIC VECTOR FIELDS ON A CR-SUBMANIFOLD OF A PARA-KÄHLERIAN MANIFOLD
}

\author{
ADELA MIHAI and RADU ROSCA
}

Received 16 July 2003

\begin{abstract}
We deal with a CR-submanifold $M$ of a para-Kählerian manifold $\widetilde{M}$, which carries a $J$-skewsymmetric vector field $X$. It is shown that $X$ defines a global Hamiltonian of the symplectic form $\Omega$ on $M^{\top}$ and $J X$ is a relative infinitesimal automorphism of $\Omega$. Other geometric properties are given.
\end{abstract}

2000 Mathematics Subject Classification: 53C15, 53C20, 53C21.

1. Introduction. CR-submanifolds $M$ of some pseudo-Riemannian manifolds $\widetilde{M}$ have been first investigated by Rosca [10], and also studied in [2, 3, 11].

If $\widetilde{M}$ is a para-Kählerian manifold, it has been proved that any coisotropic submanifold $M$ of $\widetilde{M}$ is a CR-submanifold (such CR-submanifolds have been denominated CICRsubmanifolds [6]).

In this note, one considers a foliate CICR-submanifold $M$ of a para-Kählerian manifold $\widetilde{M}(J, \widetilde{\Omega}, \tilde{g})$. It is proved that the necessary and sufficient condition in order that the leaf $M^{\top}$ of the horizontal distribution $D^{\top}$ on $M$ carries a $J$-skew-symmetric vector field $X$, that is, $\nabla X=X \wedge J X$, is that the vertical distribution $D^{\perp}$ on $M$ is autoparallel.

In this case, $M$ may be viewed as the local Riemannian product $M=M^{\top} \times M^{\perp}$, where $M^{\top}$ is an invariant totally geodesic submanifold of $M$ and $M^{\perp}$ is an isotropic totally geodesic submanifold.

Furthermore, if $\Omega$ is the symplectic form of $M^{\top}$, it is shown that $X$ is a global Hamiltonian of $\Omega$ and $J X$ is a relative infinitesimal automorphism of $\Omega$ (a similar discussion can be made for proper CR-submanifolds of a Kählerian manifold).

2. Preliminaries. Let $\widetilde{M}(J, \widetilde{\Omega}, \widetilde{g})$ be a $2 m$-dimensional para-Kählerian manifold, where, as is well known [7], the triple $(J, \widetilde{\Omega}, \tilde{g})$ of tensor fields is the paracomplex operator, the symplectic form, and the para-Hermitian metric tensor field, respectively.

If $\tilde{\nabla}$ is the Levi-Civita connection on $\widetilde{M}$, these manifolds satisfy

$$
J^{2}=I d, \quad d \widetilde{\Omega}=0, \quad(\tilde{\nabla} J) \tilde{Z}=0, \quad \tilde{Z} \in \Gamma T \widetilde{M} .
$$

Let $x: M \rightarrow \widetilde{M}$ be the immersion of an $l$-codimensional submanifold $M, l<m$, in $\widetilde{M}$ and let $T_{p}^{\perp} M$ and $T_{p} M$ be the normal space and the tangent space at each point $p \in M$. 
If $J\left(T_{p}^{\perp} M\right) \subset T_{p} M$, then $M$ is said to be a coisotropic submanifold of $\widetilde{M}$ (see [2]). If $\widetilde{W}=\operatorname{vect}\left\{h_{a}, h_{a^{*}} ; a=1, \ldots, m, a^{*}=a+m\right\}$ is a real Witt vector basis on $\widetilde{M}$, one has

$$
\tilde{g}\left(h_{a}, h_{b}\right)=\tilde{g}\left(h_{a^{*}}, h_{b^{*}}\right)=\delta_{a b} .
$$

Next, if $\widetilde{W}^{*}=\left\{\omega^{a}, \omega^{a^{*}}\right\}$ denotes the associated cobasis of $\widetilde{W}$, then $\widetilde{g}$ and $\widetilde{\Omega}$ are expressed by

$$
\begin{aligned}
& \tilde{g}=2 \sum \omega^{a} \otimes \omega^{a^{*}}, \\
& \widetilde{\Omega}=\sum \omega^{a} \wedge \omega^{a^{*}} .
\end{aligned}
$$

We recall also that $\widetilde{W}$ may split as

$$
\widetilde{W}=\widetilde{S}+\widetilde{S}^{*}
$$

where the pairing $\left(\tilde{S}, \tilde{S}^{*}\right)$ defines an involutive automorphism of square 1 , that is,

$$
J h_{a}=h_{a^{*}}, \quad J h_{a^{*}}=h_{a},
$$

and the local connection forms $\widetilde{\theta}_{B}^{A} \in \Lambda^{1} \widetilde{M}, A, B \in\{1,2, \ldots, 2 m\}$ satisfy

$$
\tilde{\theta}_{b}^{a^{*}}=0, \quad \tilde{\theta}_{b^{*}}^{a}=0, \quad \tilde{\theta}_{b}^{a}+\tilde{\theta}_{a^{*}}^{b^{*}}=0 .
$$

It has been proved in [10] that any coisotropic submanifold $M$ of a para-Kählerian manifold $\widetilde{M}$ is a CR-submanifold of $\widetilde{M}$ and such a submanifold has been called a CICRsubmanifold [6].

Let $D^{\top}: p \rightarrow D_{p}^{\top}=T_{p} M \backslash J\left(T_{p}^{\perp} M\right)$ and $D^{\perp}: p \rightarrow D_{p}^{\perp}=J\left(T_{p}^{\perp} M\right) \subset T_{p} M$ be the two complementary differentiable distributions on $M$. One has

$$
J D_{p}^{\top}=D_{p}^{\top}, \quad J D_{p}^{\perp}=T_{p}^{\perp} M,
$$

and $D^{\top}$ (resp., $D^{\perp}$ ) is called the horizontal (resp., vertical) distribution on $M$.

As in the Kählerian case, the vertical distribution $D^{\perp}$ is always involutive.

If $M$ is defined by the Pfaffian system

$$
\omega^{r}=0, \quad r=2 m+1-l, \ldots, 2 m
$$

then one has

$$
\begin{aligned}
& D_{p}^{\top}=\left\{h_{i}, h_{i^{*}}, i=1, \ldots, m-l, i^{*}=i+m\right\}, \\
& D_{p}^{\perp}=\left\{h_{r}, r=m-l+1, \ldots, m\right\} .
\end{aligned}
$$

Further denote by

$$
\varphi^{\perp}=\omega^{m-l+1} \wedge \cdots \wedge \omega^{m}
$$

the simple unit form which corresponds to $D^{\perp}$. 
Then, in order that the distribution $D^{\top}$ be also involutive, it is necessary and sufficient that $\varphi^{\perp}$ be a conformal integral invariant of $D^{\top}$, that is,

$$
\mathscr{L}_{D^{\top}} \varphi^{\perp}=f \varphi^{\perp}
$$

for a certain scalar function $f$.

By a standard calculation, one derives that the above equation implies

$$
\theta_{i}^{r}=0
$$

and in this case, one may write

$$
d \varphi^{\perp}=-\left(\sum \theta_{r}^{r}\right) \wedge \varphi^{\perp}
$$

that is, $\varphi^{\perp}$ is exterior recurrent.

In this case, as is known [2, 10], $M$ is a foliated CR-submanifold of $\widetilde{M}$.

We will investigate now the case when the leaf $M^{\top}$ of $D^{\top}$ carries a $J$-skew-symmetric vector field $X$, that is,

$$
\nabla X=X \wedge J X
$$

One may express $\nabla X$ as

$$
\nabla X=(J X)^{b} \otimes X-X^{b} \otimes J X
$$

where

$$
X=X^{i} h_{i}+X^{i^{*}} h_{i^{*}}=X^{i} \omega^{i^{*}}+X^{i^{*}} \omega^{i} .
$$

Recalling Cartan structure equations [4],

$$
\begin{aligned}
\nabla h & =\theta \otimes e \in A^{1}(M, T M), \\
d \omega & =-\theta \wedge \omega, \\
d \theta & =-\theta \wedge \theta+\Theta .
\end{aligned}
$$

In the above equations, $\theta$, respectively $\Theta$, are the local connection forms in the bundle $W$, respectively the curvature forms on $M$.

Then making use of Cartan structure equations, one finds by a standard calculation that (2.16) implies that the vertical distribution $D^{\perp}$ is autoparallel, that is, $\nabla_{Z^{\prime}} Z^{\prime \prime} \in D^{\perp}$, for all $Z^{\prime}, Z^{\prime \prime} \in D^{\perp}$, which, in terms of connection forms, is expressed by

$$
\theta_{r}^{i}=0
$$

We agree to call $\theta_{r}^{i}$ and $\theta_{i}^{r}$ the mixed connection forms.

Taking account of (2.13) and (2.19), one derives from (2.16)

$$
d X^{b}=2(J X)^{b} \wedge X^{b},
$$

which agrees with the general equation of skew-symmetric killing vector fields [5, 8]. 
Next, by (2.1), one has

$$
\nabla J X=(J X)^{b} \otimes J X-X^{b} \otimes X
$$

which shows that $J X$ is a gradient vector field.

Hence, we may state the following theorem.

THEOREM 2.1. Let $x: M \rightarrow \widetilde{M}$ be an improper immersion of a CR-submanifold in a para-Kählerian manifold $\widetilde{M}(J, \widetilde{\Omega}, \widetilde{g})$ and let $D^{\top}$ (resp., $\left.D^{\perp}\right)$ be the horizontal distribution (resp., the vertical distribution) on M. If $M$ is a foliate CR-submanifold, then the necessary and sufficient condition in order that the leaf $M^{\top}$ of $D^{\top}$ carries a J-skew-symmetric vector field $X$ is that $D^{\perp}$ is an autoparallel foliation. In this case, the CR-submanifold $M$ under consideration may be viewed as the local Riemannian product $M=M^{\top} \times M^{\perp}$, where $M^{\top}$ is an invariant totally geodesic submanifold of $M$ and $M^{\perp}$ is an isotropic totally geodesic submanifold. In addition, in this case, $J X$ is a gradient vector field.

3. Properties. In this section, we will pointout some additional properties of $X$ involving the symplectic form $\Omega$ of $M^{\top}$ and the exterior covariant differential $d^{\nabla}$ of $\nabla X$. Operating on (2.16) and (2.21), one derives by a short calculation

$$
\begin{aligned}
d^{\nabla}(\nabla X) & =\nabla^{2} X=2\left(X^{b} \wedge(J X)^{b}\right) \otimes J X, \\
d^{\nabla}(\nabla J X) & =\nabla^{2} J X=2\left(X^{b} \wedge(J X)^{b}\right) \otimes X,
\end{aligned}
$$

which gives

$$
\begin{aligned}
& \nabla^{2}(X+J X)=2\left(X^{b} \wedge(J X)^{b}\right) \otimes(X+J X), \\
& \nabla^{2}(X-J X)=-2\left(X^{b} \wedge(J X)^{b}\right) \otimes(X-J X) .
\end{aligned}
$$

Therefore, we agree to define $X+J X$ and $X-J X$ as 2-covariant recurrent vector fields. It should also be noticed that by reference to the general formula

$$
\nabla_{V}\left(X_{1} \wedge \cdots \wedge X_{p}\right)=\sum\left(X_{1} \wedge \cdots \wedge \nabla_{V} X_{j} \wedge \cdots \wedge X_{p}\right), \quad V \in \Gamma T M
$$

one finds by (2.15) and (2.21)

$$
\nabla_{V}(X \wedge J X)=2 g(V, J X)(X \wedge J X)
$$

This shows that the covariant derivative of $X \wedge J X$ with respect to any vector field $V$ is proportional to $X \wedge J X$.

On the other hand, by the general formula

$$
\nabla^{2} V\left(Z, Z^{\prime}\right)=R\left(Z, Z^{\prime}\right) V
$$


where $R$ denotes the curvature tensor field and $V, Z, Z^{\prime}$ are vector fields, one has (see also [9])

$$
\mathscr{R}(Z, V)=\operatorname{Tr} R(\cdot, Z) V
$$

where $\mathscr{R}$ is the Ricci tensor field of $\nabla$.

Since in the case under consideration one must take in (3.6) the para-Hermitian trace, then setting in (3.6) $Z=V=X$, one finds

$$
\mathscr{R}(X, X)=0
$$

that is, the Ricci curvature of $X$ vanishes.

Denote by $\widetilde{\Omega}$ the symplectic form of $\widetilde{M}$, then $\Omega=\left.\widetilde{\Omega}\right|_{M^{\top}}$ is a symplectic form of rank equal to the dimension of $M^{\top}$, that is, in our case, $2(m-l)$.

Then, if ${ }^{b} Z: Z \rightarrow-i_{Z} \Omega$ is the symplectic isomorphism, by a short calculation and on behalf of (2.4), one gets

$$
{ }^{b} X=-(J X)^{b},
$$

and since $J X$ is a gradient vector field, we conclude according to a known definition (see also [1]) that $X$ is a global Hamiltonian of $\Omega$.

In a similar manner, one finds

$$
{ }^{b}(J X)=X^{b},
$$

and by (2.20), it follows that

$$
d\left(\mathscr{L}_{J X} \Omega\right)=0
$$

which shows that $J X$ is a relative infinitesimal automorphism of $\Omega$ [1].

We state the following theorem.

THEOREM 3.1. Let $M$ be a CR-submanifold of a para-Kählerian manifold $\widetilde{M}$ and let $\Omega$ be the symplectic form on $M^{\top}$. If $M$ carries a J-skew-symmetric vector field $X$, then the following properties hold:

(i) $X$ is a global Hamiltonian of $\Omega$ and $J X$ is a relative infinitesimal automorphism of $\Omega$;

(ii) the Ricci tensor field $\mathscr{R}(X, X)$ vanishes;

(iii) the vector fields $X+J X$ and $X-J X$ are 2-covariant recurrent.

ACKNOWLEDGMENT. The first author was supported by a Japan Society for the Promotion of Science Postdoctoral Fellowship.

\section{REFERENCES}

[1] R. Abraham, J. E. Marsden, A. Kelley, and A. N. Kolmogorov, Foundations of Mechanics. A Mathematical Exposition of Classical Mechanics with an Introduction to the Qualitative Theory of Dynamical Systems and Applications to the Three-Body Problem, W. A. Benjamin, New York, 1967. 
[2] K. Buchner and R. Rosca, Co-isotropic submanifolds of a para-co-Kählerian manifold with concircular structure vector field, J. Geom. 25 (1985), no. 2, 164-177.

[3] M. T. Calapso and R. Rosca, Cosymplectic quasi-Sasakian pseudo-Riemannian manifolds and coisotropic foliations, Rend. Circ. Mat. Palermo (2) 36 (1987), no. 3, 407-422.

[4] É. Cartan, Les Systèmes Différentiels Extérieurs et Leurs Applications Géométriques, Actualités Sci. Ind., no. 994, Hermann et Cie., Paris, 1945.

[5] F. Defever, R. Rosca, and L. Verstraelen, On null Killing vector fields, Riv. Mat. Univ. Parma (5) 1 (1992), 239-253.

[6] V. V. Goldberg and R. Rosca, Contact co-isotropic CR submanifolds of a pseudo-Sasakian manifold, Int. J. Math. Math. Sci. 7 (1984), no. 2, 339-350.

[7] P. Libermann, Sur le problème d'équivalence de certaines structures infinitésimales, Ann. Mat. Pura Appl. (4) 36 (1954), 27-120 (French).

[8] I. Mihai, R. Rosca, and L. Verstraelen, Some Aspects of the Differential Geometry of Vector Fields, Centre for Pure and Applied Differential Geometry (PADGE), vol. 2, Katholieke Universiteit Brussel Group of Exact Sciences, Brussels, 1996.

[9] W. A. Poor, Differential Geometric Structures, McGraw-Hill, New York, 1981.

[10] R. Rosca, CR-sous-variétés co-isotropes d'une variété parakählérienne, C. R. Acad. Sci. Paris Sér. I Math. 298 (1984), no. 7, 149-151 (French).

[11]_ Variétés neutres $\tilde{M}$ admettant une structure conforme symplectique et feuilletage coisotrope, C. R. Acad. Sci. Paris Sér. I Math. 300 (1985), no. 18, 631-634 (French).

Adela Mihai: Department of Geometry, Faculty of Mathematics, University of Bucharest, Street Academiei 14, 70109 Bucharest, Romania

E-mail address: ade 1a@math.math.unibuc. ro

Radu Rosca: 59 avenue Emile Zola, 75015 Paris, France 


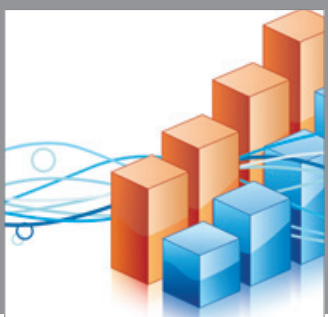

Advances in

Operations Research

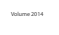

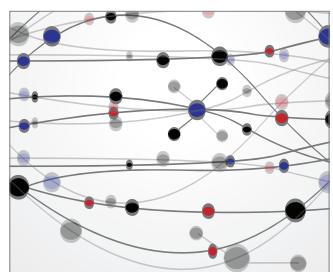

\section{The Scientific} World Journal
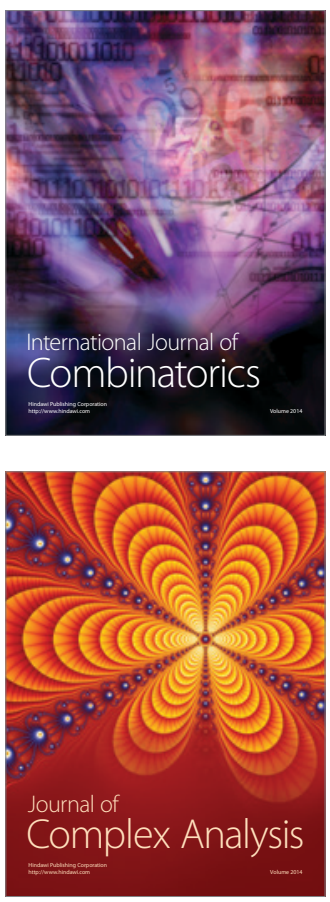

International Journal of

Mathematics and

Mathematical

Sciences
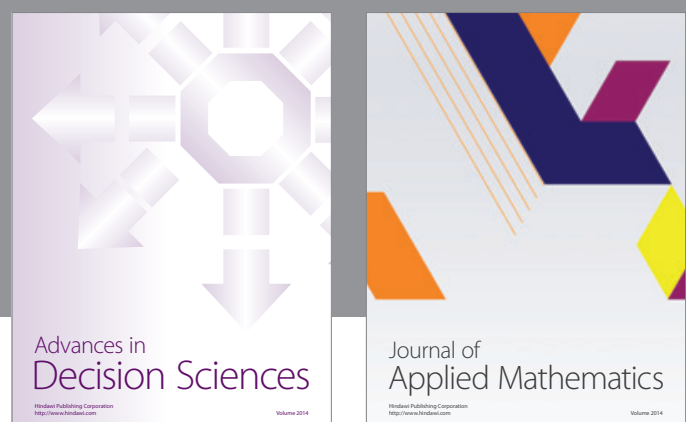

Journal of

Applied Mathematics
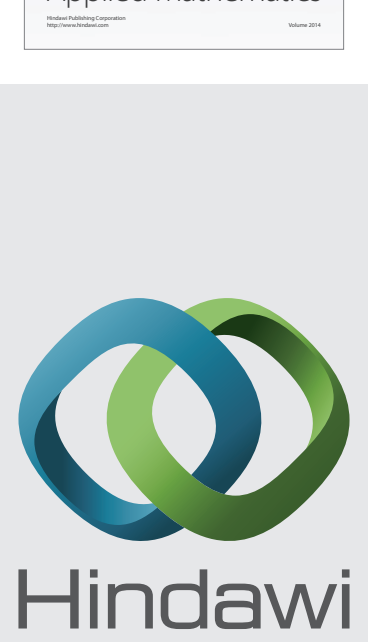

Submit your manuscripts at http://www.hindawi.com
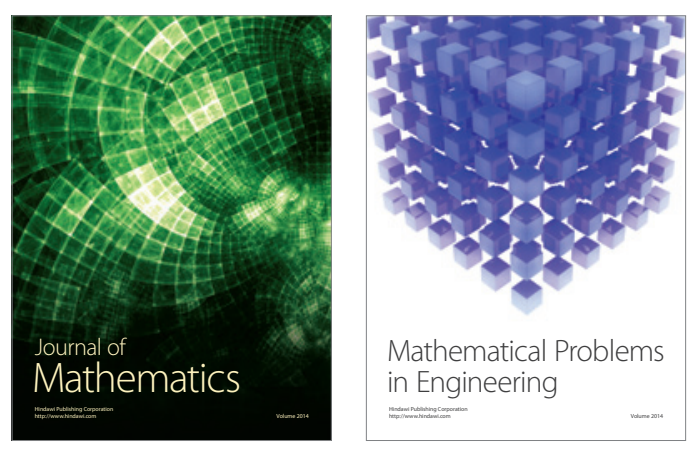

Mathematical Problems in Engineering
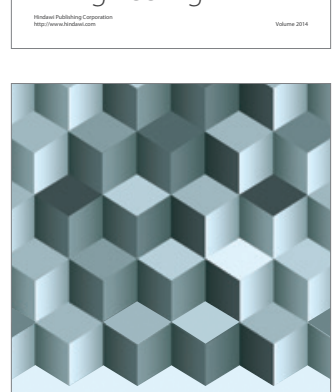

Journal of

Function Spaces
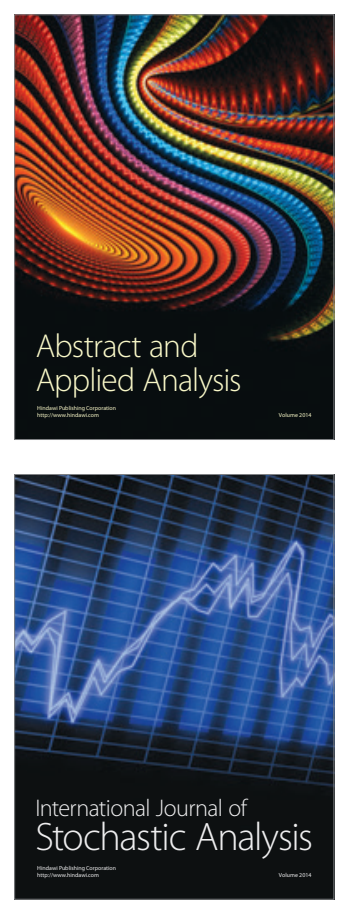

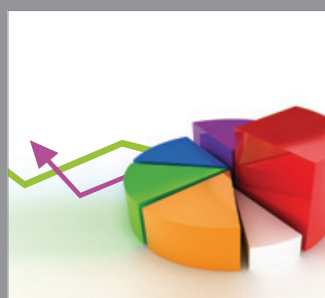

ournal of

Probability and Statistics

Promensencen
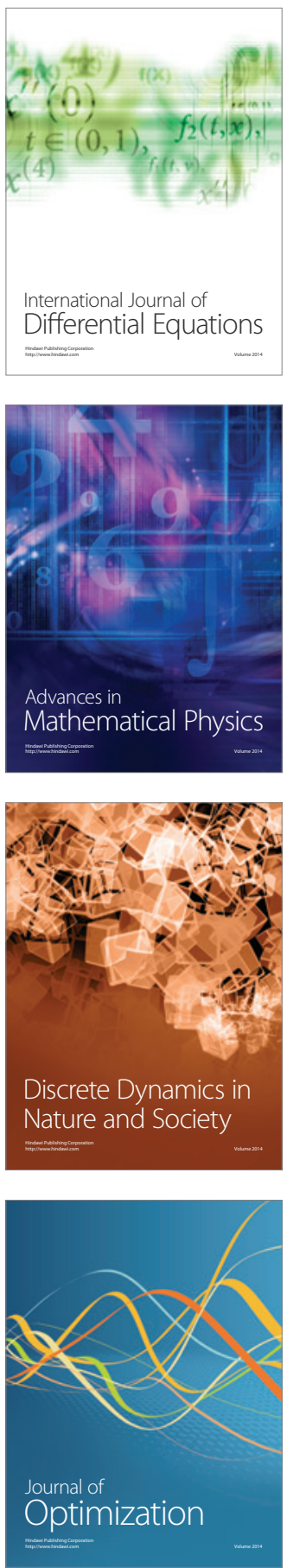